\title{
Dexmethylphenidate hydrochloride in the treatment of attention deficit hyperactivity disorder
}

\author{
Feng Liu \\ Haruka Minami \\ Raul R Silva \\ New York University School of \\ Medicine, Division of Child and \\ Adolescent Psychiatry, New York, \\ NY, USA
}

Correspondence: Raul R Silva NY School of Medicine, Department of Psychiatry, 550 First Avenue, NB2 IS6,

New York, NY 10016 , USA

Tel + I 2I 22636602

Fax + I 2122630202

Email raul.silva@med.nyu.edu

\begin{abstract}
Attention-deficit/hyperactivity disorder (ADHD) affects a large number of children. For decades, the stimulants have been the mainstay of pharmacological treatment for ADHD. Dexmethylphenidate ( $d$-MPH), the $d$-isomer of the traditional racemic mixtures of $d$,l-threo$(R, R)-\mathrm{MPH}$, was recently introduced as another potential option in the stimulant class of medications. This paper reviews and summarizes the available research literature on $d$-MPH regarding pharmacodynamic, pharmacokinetic, chemical structure, receptor binding, toxicology, and clinical perspectives. $d$-MPH potentially may offer some advantages in the realms of absorption and duration of action compared with its racemic counterpart. The differences in pharmacokinetics and clinical implications of the immediate-release and extended-release forms of $d$-MPH are also compared and contrasted.
\end{abstract}

Keywords: ADHD, dexmethylphenidate, chemistry, receptor binding, toxicology, pharmacokinetics

\section{Brief history}

Attention-deficit/hyperactivity disorder (ADHD) affects a large number of children. In the United States, rates range from $5 \%$ to $6 \%$ of school-age children (Guevara et al 2002; Lesesne et al 2003). The illness is characterized by a triad of symptom clusters which include inattention, hyperactivity, and impulsivity. Usually the illness is first detected during the early school years (Goldman et al 1998), but can persist during adolescence and even adulthood. If left untreated, ADHD can be associated with a number of poor outcomes including academic failure, delinquency, and problems with substance abuse (Barkley 1990; Biederman et al 1996; Dulcan 1997; Wilens et al 2004).

Stimulants have been the mainstay of pharmacological treatment of ADHD for over 60 years. Their effect on disruptive behavior was discovered in 1937, when these drugs proved to increase compliance, improve academic performance, and reduce motor activity in hyperactive children (Bradley 1937). Methylphenidate (MPH) is the most frequently prescribed among stimulant agents. It has proven efficacy on ADHD symptoms (Kimko et al 1999; Greenhill et al 2002).

\section{d-MPH vs d,I-MPH (racemic MPH)}

Until the introduction of the $d$-MPH $(d$-threo- $(R, R)-\mathrm{MPH}, d-\mathrm{MPH})$ in 2002 , all clinically used MPH formulations contained a racemic $(1: 1)$ mixture of $d$-threo- $(R, R)$ MPH and l-threo-(R,R)-MPH isomers (Figure 1). The development of $d$-MPH was based on the findings that similar improvement on sustained attention was achieved after treatment with $d$-equivalent doses of $d$-MPH and $d, l-\mathrm{MPH}$, but not after $l$-MPH (Srinivas et al 1992). It was found that clinical efficacy was highly correlated with 


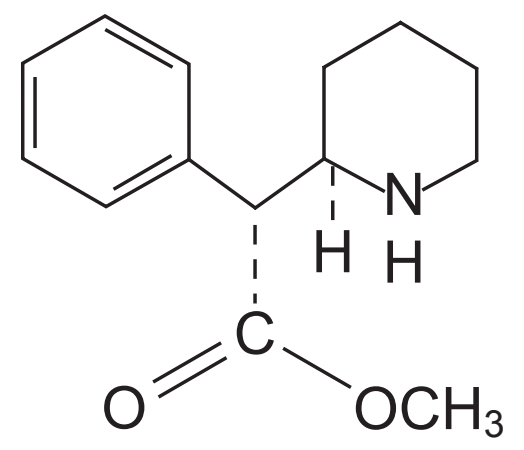

d-threo-methylphenidate

Figure I Chemical structures of $d$ - and l-threo-methylphenidate.

plasma concentrations of $d$-MPH. The elimination of the $l$-isomer does not diminish the efficacy of an acute dose of MPH (Quinn et al 2004). The efficacy of the $d$-isomer was equivalent to the racemic preparation in ameliorating the target symptoms of ADHD and increasing academic productivity. Thus, it was thought that the efficacy of MPH resides in the $d$-isomer (Lim et al 1986; Patrick et al 1987; Srinivas et al 1987, 1992). Other research demonstrated that besides clinical effectiveness, the undesired pressor and anorexic actions of $d, l-\mathrm{MPH}$ also appear to reside in the $d$-isomer (Srinivas et al 1992; Anonymous 2002; Scheffler et al 2003; Teo et al 2004).

$d$-MPH not only appears to be the more active of the two enantiomers, but also better absorbed; plasma concentrations of $d$-MPH are many times higher than the $l$-enantiomer after oral dosing of racemic MPH (Hubbard et al 1989; Ding et al 1997; Kimko et al 1999; Modi et al 2000) or intravenous administration (Kimko et al 1999). This is seen with both immediate-release (Kimko et al 1999) and sustained-released (Hubbard et al 1989; Kimko et al 1999) forms of racemic MPH. The pre-systemic metabolism and metabolic clearance of $d, l-\mathrm{MPH}$ is also an enantioselective process which, too, contributes to markedly higher plasma concentrations of $d$-MPH relative to $l$-MPH (Markowitz et al 2003; Patrick et al 2005). It was also found that equimolar doses of $d$-MPH yielded similar pharmacokinetics as those noted with administration of the $d$,l-racemic mixture (Srinivas et al 1992).

As a result, the $d$-MPH product was developed, and approved by the FDA for clinical use on August 312001. The $d$-MPH product appears to double the therapeutic

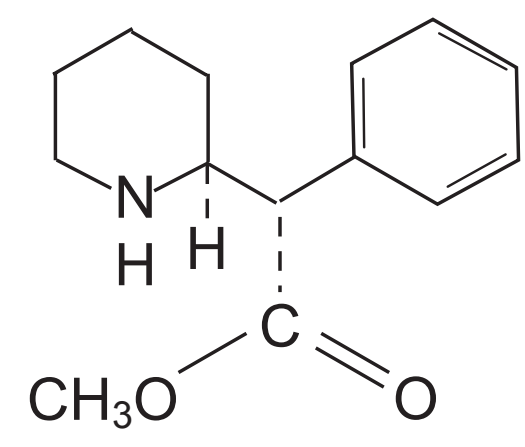

I-threo-methylphenidate

potency of the racemic $d, l-\mathrm{MPH}$; when only half dose of racemic $d, l$-MPH was used, $d$-MPH produced similar pharmacokinetic parameters $\left(\mathrm{C}_{\max }, \mathrm{T}_{\max }\right.$, and half-life $)$ (Anonymous 2002; Keating and Figgitt 2002; Markowitz et al 2003). The administration of $d$-MPH with food has no significant effect on bioavailability, but with racemic MPH, $\mathrm{T}_{\max }$ was delayed by 1 hour (Teo et al 2004). In addition, a number of studies (Patrick et al 1981; Arnold et al 2004; Quinn et al 2004; Silva et al 2004; Wigal et al 2004) suggested that $d$-MPH might have a longer duration of action than $d$,l-MPH on tests measuring attention, hyperactivity, and impulsivity. However, this requires further controlled study. As mentioned before, the use of $d-\mathrm{MPH}$ in ADHD allows the administration of lower doses than those currently prescribed with $d, l-\mathrm{MPH}$.

\section{Chemistry}

Molecules that are non-superimposable mirror images of one another are termed enantiomers and when equal amounts (1:1) of enantiomeric molecules are present together, the product is termed racemic. Most current commercial formulations of MPH consist of the racemic mixture of the threo pair of MPH isomers (d,l-threo $\mathrm{MPH})$.

Although MPH has two chiral centers, and therefore four isomers, a d,l-erythro-MPH pair and a d,l-threo-MPH pair, only the racemic mixture of the threo pair of enantiomers (d,l-threo-MPH) is used therapeutically. This is due to the recognition that it has fewer side-effects than the erythro enantiomers. Figure 1 shows the chemical structures of $d$ - and $l$-threo-MPH. It should be noted that the phenethylamine moiety of $d$-MPH is shared by dopamine 
and norepinephrine. It is the phenethylamine structure that provides the transporter receptor affinity and it competes with dopamine for binding (Kimko et al 1999).

The predominant metabolic pathway of MPH is de-esterification to form pharmacologically inactive $d$ - or $l$-ritalinice acid by the carboxyesterase-I isoform, CES1A1 (Sun et al 2004). It was shown that $d$,l-threomethylphenidate was stereoselectively metabolized in humans (Lim et al 1986; Srinivas et al 1987), with l-threoMPH being eliminated faster than $d$-threo-MPH (Srinivas et al 1992; Sun et al 2004). In a double-blind, four-way, randomized, crossover study, $l$-MPH showed no difference vs placebo in improving the sustained attention in humans. Moreover, a 5-mg dose of $d$-threo-MPH has the same attention improvement effect as a 10-mg dose of d,l-threo-MPH racemic mixture. Hence, the clinical effect of MPH is attributed to the $d$-enantiomer (Srinivas et al 1992). The brand name for $d$-MPH is Focalin ${ }^{\circledR}$.In terms of its chemical composition, $d$-MPH hydrochloride is methyl $\alpha$-phenyl-2-piperidineacetate hydrochloride, (R, $\left.\mathrm{R}^{\prime}\right)-(+)-$. Its empirical formula is $\mathrm{C}_{14} \mathrm{H}_{19} \mathrm{NO}_{2} \cdot \mathrm{HCl}$. Its molecular weight is 269.77 .

\section{Binding and imaging studies of $d$-isomer vs l-isomer: from animals to humans}

Although its mechanism is poorly understood, pharmacodynamic studies have suggested that the therapeutic effect of MPH in the treatment of ADHD appears to lie primarily in increasing the synaptic concentration of dopamine by blocking dopamine transporters (DAT) (Gatley et al 1996; Ding et al 1997; Davids et al 2002), as well as in blocking norepinephrine transporters. This is in agreement with recent reports that there is significantly increased expression of DAT in ADHD patients (Volkow et al 2002) and the effects of MPH administration is associated with a decrease in DAT receptor concentration in the striatum (Krause et al 2000).

Normally, DAT decreases with age (Volkow et al 1996). However, in methamphetamine abusers, the number of DAT also are reduced (Volkow et al 2001a, 2001b). Recovery of DAT receptors is evident with protracted abstinence (Volkow et al 2001a). The binding of $d$-MPH to DAT in the striatum is reduced by cocaine in a dose-dependent manner (Fowler et al 1998).

Studies in humans, baboons, and rats seem to indicate that the pharmacological specificity of MPH resides predominantly in its $d$-isomer (Srinivas et al 1992; Ding et al 1997; Anonymous 2002). Thus, in vivo binding measures of radiolabeled $d$-MPH to dopamine transporter in the brain with positron emission tomography (PET) or microdialysis have not only become a valuable technique to analyze pharmacodynamics of MPH and its enantiomers (Ding et al 1995, 1997, 2004; Gatley et al 1995; Logan et al 1996, 2001; Volkow et al 2002; Kilbourn 2004), but also served as an effective tool to assess expression or density of dopamine transporters in tissues of humans and animals (Volkow et al 1995, 1996b, 2001a, 2001b; Fowler et al 1998; Gatley et al 1999).

PET studies provide a quantitative measure of distribution volume ratios, which represent specific radioligand binding (Volkow et al 1995; Wang et al 1995). Measures of distribution volume ratios are based upon the following assumptions: (i) Even if the plasma concentration of the radiolabeled tracer is unknown (for example, without blood samples from patients), "non-invasive" quantification is still possible using a reference region with graphical analysis (Logan et al 1996); (ii) for neuroreceptor-transporter modeling, no specific binding occurs in the reference region (Volkow et al 2002); (iii) the distribution volume of the reference region is the same as the distribution volume of the first compartment (free and non-specifically bound tracer) in the target region. Since the basal ganglia of striatum expresses more dopamine transporters (DAT), it is considered as the target region for $d-\mathrm{MPH}$, while the cerebellum does not express DAT, so it usually is considered the reference region (Volkow et al 2002; Kilbourn 2004). Thus, the tissue distribution volume ratio of $\left[{ }^{11} \mathrm{C}\right] d-\mathrm{MPH}$ in the basal ganglia containing the specific binding to that in the cerebellum which demonstrates non-specific binding should be considered to be a reflection of dopamine transporter availability.

Recent results from PET and other imaging studies for the two enantiomers of racemic MPH demonstrated that $\left[{ }^{11} \mathrm{C}\right] d$-MPH bound to the dopamine transporter in the basal ganglia is highly specific and reproducible (Ding et al 1997). However, there is no evidence of regional specific binding for $\left[{ }^{11} \mathrm{C}\right]$ l-threo-MPH in the brain. These results suggest that the binding of MPH to DAT in the striatum is enantiospecific. These data are also in agreement with those from basic and clinical studies showing that $d$-MPH at therapeutic doses exerts much stronger effects than racemic MPH or l-threoMPH on extracellular dopamine in rats and on improvement in sustained attention in children (Srinivas et al 1992; Patrick et al 1987). 
Moreover, $d$ - and $l$-MPH also undergo enantioselective metabolism (Srinivas et al 1987; Hubbard et al 1989; Anonymous 2002; Sun et al 2004), as evidenced by the following findings. First, as mentioned before, because of rapid pre-systemic metabolism, orally administered l-threoMPH is poorly absorbed in humans. Thus, the absolute bioavailability of $d$-MPH is significantly greater than that of l-threo-MPH (Srinivas et al 1993; Sun et al 2004). Second, because there is a correlation between plasma concentrations and DAT occupancy, the plasma concentration could serve as an indicator for DAT binding (Srinivas et al 1992). However, DAT binding by $d$-MPH could also be obtained from calculated tissue distribution volume without patients' blood samples (Logan et al 1996). Third, while the rate of brain uptake for both enantiomers was not significantly different, the rate of clearance after 1.5 hours from the brain or plasma was much slower for $d$-MPH than that of l-threoMPH (Volkow et al 2002). Fourth, greater than 50\% DAT binding in the basal ganglia by $d$-MPH may be required for therapeutic efficacy for ADHD. However, despite greater than $50 \%$ DAT occupancy by $d$-MPH when administered intravenously or orally, the reinforcing effects were not perceived by patients receiving oral $d$-MPH (Volkow et al 2002). This phenomenon is possibly due to slower blockade of DAT by oral $d$-MPH and may explain why intravenous injection of MPH may lead to addiction. Finally, there is no inter-conversion between the enantiomers after oral administration of pure $d$ - and $l$-MPH separately (Volkow et al 2002).

The recent in vitro binding study of Markowitz et al (2005) found that $d$-MPH exhibited prominent effects at the norepinephrine transporter site, even exceeding the activity at the DAT. The result demonstrated that affinity for catecholaminergic sites largely resides in the $d$-isomer.

\section{Toxicology studies in animals}

Animal toxicology studies showed that the toxicity of $d$-MPH and $d, l$-MPH were comparable at equimolar doses (Teo et al 2002). A recent comparative study assessing the acute behavioral toxicity of $d-\mathrm{MPH}, l-\mathrm{MPH}$, and $d, l-\mathrm{MPH}$ in rats has revealed that females were more sensitive than males to some toxic behavioral effects of the $l$-isomer and $d, l$-MPH (racemic), suggesting a possible sex difference in sensitivity (Teo et al 2003d). In related studies, increased incidence of dilated pupils and vocalization was found with $d, l-\mathrm{MPH}$ compared with the pure $d$-MPH given at half the dose (Teo et al 2002). In the same study, no other significant toxic effects were found in perinatal or postnatal rats at a dose 25 times the maximum daily human therapeutic dose (Teo et al 2002). Another toxicology study in beagle dogs demonstrated that only minimal reversible toxicity such as reduced appetite and weight loss were found in male and female dogs receiving $10 \mathrm{mg} / \mathrm{kg}$ of $d$-MPH and $20 \mathrm{mg} / \mathrm{kg}$ of $d, l$-MPH for 90 days (Teo et al 2003c).

Neither $d$-MPH nor $d, l$-MPH were found to be carcinogenic or clastogenic in either in vitro or in vivo studies, although very high oral doses of the $l$-MPH produce behavioral, and even lethal, effects in mice (Teo et al 2003a). The authors believed that $d-\mathrm{MPH}$ and $d, l-\mathrm{MPH}$ do not present carcinogenic risk to humans. Animal studies in rats and rabbits also suggested that $d$-MPH and $d, l$-MPH were not teratogenic (Teo et al 2003b). The same study suggested that some negative behaviors in pregnant animals may be attributed to $l$-MPH because inclusion of the $l$-isomer, when comparing $d, l$-MPH and $d$-MPH at half the dose, resulted in repetitive pawing, dilated pupil, and aggressive behavior. A recent study, using PET and MicroPET to trace orally administered, as well as radiolabeled $d$ - and $l$-MPH, in the brain of baboons and rats, suggested that $l$-MPH may not be pharmacologically active. Additionally, $l$-MPH might contribute to the behavioral and side-effect profiles of $d, l$-MPH during the treatment of ADHD (Ding et al 2004).

\section{Immediate-release and extended- release form of $\mathrm{d}-\mathrm{MPH}$}

The immediate-release (IR) $d$-MPH product is deemed to provide effective management of ADHD at half the dose of racemic MPH products and was shown to be effective and well tolerated in clinical trials (Swanson et al 2002; Arnold et al 2004; Quinn et al 2004; Wigal et al 2004). An initial pharmacokinetic study showed that after an oral dose of $d$ $\mathrm{MPH}$, plasma $d$-MPH concentration increased rapidly, reaching maximum levels at $1-1.5$ hours post-dose in fasting states. Plasma pharmacokinetics of $d$-MPH were similar to $d, l$-MPH given the equimolar amounts of $d$-MPH. The mean plasma elimination half-life for $d$-MPH is approximately 2.2 hours (Anonymous 2002). However, recent studies, including one post hoc analysis (Weiss et al 2004), one open-labeled (Silva et al 2004), and three placebo-controlled clinical studies (Arnold et al 2004; Quinn et al 2004; Wigal et al 2004) in children with ADHD, suggested that IR $d$-MPH may produce a longer duration of therapeutic action ( $\geq 6$ hours) which cannot be explained solely on the basis of the agent's pharmacokinetics. 
Some authors have postulated that the need for a midday dose for IR MPH products may erode social functioning and sometimes the self-esteem of children with ADHD. Thus, an extended-release form of $d$-MPH ( $d$-MPH-XR) was designed, known as Focalin $\mathrm{XR}^{\circledR}$, and approved by FDA for clinical use in May 2005. This product is a modified release capsule formulation of $d$-MPH specifically engineered to deliver a bimodal (biphasic) release of $d$-MPH that mimics a twice daily $d$-MPH dosing (ie, two distinct peaks approximately 4 hours apart). The $d$-MPH-XR product uses the same proprietary SODAS (Spheroidal Oral Drug Absorption System) technology as one of the racemic MPH products (Ritalin-LA ${ }^{\circledR}$ ) currently on the market. For each of these products, capsules are filled with a mixture of $50 \%$ immediate-release beads and $50 \%$ enteric-coated, delayed-release beads, thus providing immediate release and a second delayed-release component. After administration of $d$-MPH-XR, the first peak concentration $\left(\mathrm{C}_{\max }\right)$ of $d$-MPH occurs on average after 1.5 hours (typical range $1-4$ hours). The mean time to the inter-peak minimum is slightly shorter, and time to the second peak $\left(\mathrm{t}_{\max }\right)$ is slightly longer for $d$-MPH- XR given once daily (about 6.5 hours, range $4.5-7$ hours) compared with the IR $d$-MPH given in two doses 4 hours apart. The $d$-MPH-XR product given once daily exhibits a lower second peak concentration $\left(\mathrm{C}_{\max 2}\right)$, higher inter-peak minimum concentrations, and fewer peak and trough fluctuations than the IR form of the agent, given in two doses 4 hours apart. The area under the plasma concentration-time curve (AUC) after administration of $d$-MPH-XR given once daily is equivalent to the same total dose of IR $d$-MPH tablets given in two doses 4 hours apart (Novartis Pharmaceuticals Corporation 2005).

The clinical efficacy as well as safety of $d$-MPH-XR in treating children with ADHD was demonstrated in a randomized, double-blind, placebo-controlled clinical trial (Greenhill et al 2005). In this study, flexible dosing schedules of between 5 and $30 \mathrm{mg}$ of $d$-MPH-XR were administered once daily over a 7-week period. Greenhill et al (2005) reported that $d$-MPH-XR was associated with significantly improved attention and behavior in children with ADHD. Silva et al $(2005,2006)$, in two separate studies, demonstrated that the therapeutic effect significantly differentiated from placebo during each time point of the 12-hour laboratory classroom studies. Both of these studies employed a double-blind, placebo-controlled, crossover design. The sample sizes were slightly more than 50 patients in each trial; all subjects satisfied DSM-IV criteria for ADHD.
The children ranged in age between 6 and 12 years. In both studies, the $d$-MPH-XR dose administered was $20 \mathrm{mg}$ /day. In the two studies, Swanson, Kotkin, Agler, M-Flynn, and Pelham (SKAMP) scales and math tests were administered. Significant differences were observed across all measures and in each of the subscales at each of the time points studied. Another noteworthy observation is related to the elapsed time at which efficacy was first detected. In the Silva et al (2005) study significant differences were noted at the first time point measured, which was half an hour after medication administration.

\section{Conclusions}

Based on the information we have reviewed it seems that $d$-MPH is a useful agent in the treatment of ADHD. Furthermore, it should be noted it is the only MPH product approved for the treatment of this disorder in adults. The literature reviewed identified that there are meaningful differences in the clinical efficacy and pharmacokinetic profile between the $d$ - and $l$-isomers of MPH. When comparing the single isomer with the racemic compound, a consistently noted difference across studies is that $d$-MPH has a longer duration of action than $d, l-\mathrm{MPH}$. This has been reported with both immediate-release and extended-release preparations. Further study is required to more fully understand the side-effect differences between the $d$-isomer and its racemic counterpart.

\section{Disclosures}

RRS is a consultant, has received research support, and is on the speakers bureau for Novartis Pharmaceuticals, he has also served on the Speakers Bureau of McNeil Pharmaceuticals and has received research support from Celgene Corporation. FL and HM have not conflicts of interest to report.

\section{References}

Anonymous. 2002. Dexmethylphenidate-Novartis/Celgene. Focalin, D-MPH, D-methylphenidate hydrochloride, D-methylphenidate, dexmethylphenidate, dexmethylphenidate hydrochloride. Drugs in $R \& D, 3: 279-82$.

Arnold LE, Lindsay RL, Conners CK. et al. 2004. A double-blind, placebocontrolled withdrawal trial of dexmethylphenidate hydrochloride in children with attention deficit hyperactivity disorder. J Child Adolesc Psychopharmacol, 14:542-54.

Barkley RA, 1990. Major life activity and health outcomes associated with attention-deficit/hyperactivity disorder. J Clin Psychiatry, 63(Suppl 12):10-15.

Biederman J, Faraone S, Milberger S, et al. 1996. A prospective 4-year follow-up study of attention-deficit hyperactivity and related disorders. Arch Gen Psychiatry, 53:437-46. 
Bradley C. 1937. The behavior of children receiving benzedrine. Am $J$ Psychiatry, 94:577-85.

Davids E, Zhang K, Kula NS, et al. 2002a. Effects of norepinephrine and serotonin transporter inhibitors on hyperactivity induced by neonatal 6-hydroxydopamine lesioning in rats. J Pharmacol Exp Ther, 301:1097-102.

Ding YS, Fowler JS, Volkow ND, et al. 1997. Chiral drugs: comparison of the pharmacokinetics of [11C]d-threo and 1-threo-methylphenidate in the human and baboon brain. Psychopharmacology, 131:71-8.

Ding YS, Fowler JS, Volkow ND, et al. 1995. Carbon-11-d-threomethylphenidate binding to dopamine transporter in baboon brain. J Nucl Med, 36:2298-305.

Ding YS, Gatley SJ, Thanos PK, et al. 2004. Brain kinetics of methylphenidate (Ritalin) enantiomers after oral administration. Synapse, 53:168-75.

Dulcan M. 1997. Work group on quality issues. Practice parameters for the assessment and treatment of children, adolescents, and adults with attention-deficit/hyperactivity disorder. J Am Acad Child Adolesc Psychiatry, 36:S85-121.

Fowler JS, Volkow ND, Logan J et al. 1998. Measuring dopamine transporter occupancy by cocaine in vivo: radiotracer considerations. Synapse, 28:111-16.

Gatley SJ, Ding YS, Volkow ND, et al. 1995. Binding of d-threo[11C]methylphenidate to the dopamine transporter in vivo: insensitivity to synaptic dopamine. Eur J Pharmacol, 281:141-9.

Gatley SJ, Pan D, Chen R, et al. 1996. Affinity of methylphenidate derivatives for dopamine, Norepinephrine and serotonin transporters. Life Sci, 58:231-9.

Gatley SJ, Volkow ND, Gifford AN, et al. 1999. Dopamine-transporter occupancy after intravenous doses of cocaine and methylphenidate in mice and humans. Psychopharmacology, 146:93-100.

Goldman LS, Genel M, Bezman RJ, et al, 1998: Diagnosis and treatment of attention-deficit/hyperactivity disorder in children and adolescents. Council on Scientific Affairs, American Medical Association. JAMA, 279:1100-7.

Greenhill LL, Pliszka S, Dulcan MK, et al. 2002. American Academy of Child and Adolescent Psychiatry. Practice parameter for use of stimulant medications in the treatment of children, adolescents, and adults. $J \mathrm{Am}$ Acad Child Adolesc Psychiatry, 41(Suppl 2):26-49S

Greenhill L, et al. 2005. Extended-release dexmethylphenidate in child and adolescent with ADHD. 158th Annual Meeting of the American Psychiatric Association. May 21-25, 2005; Atlanta, GA, USA.

Guevara J, Lozano P, Wickizer et al. 2002. Psychotropic medication use in a population of children who have attention-deficit/hyperactivity disorder. Pediatrics, 109:733-9.

Hubbard JW, Srinivas NR, Quinn D, et al. 1989. Enantioselective aspects of the disposition of d.1-threo-methylphenidate after the administration of a sustained-release formulation to children with attention deficithyperactivity disorder. J Pharma Science, 78:944-7.

Keating GM, Figgitt DP. 2002. Dexmethylphenidate. Drugs, 62:1899-904; discussion 1905-8.

Kilbourn MR. 2004. Long-term reproducibility of in vivo measures of specific binding of radioligands in rat brain. $\mathrm{Nucl} \mathrm{Med} \mathrm{Biol,31:591-5.}$

Kimko HC, Cross JT, Abernethy DR, et al. 1999. Pharmacokinetics and clinical effectiveness of methylphenidate. Clin Pharmacokinet, 37:457-70.

Krause KH, Dresel SH, Krause J, Kung HF, Tatsch K. 2000. Increased striatal dopamine transporter in adult patients with attention deficit hyperactivity disorder: effects of methylphenidate as measured by single photon emission computed tomography. Neurosci Lett, 285: $107-10$.

Lesesne CA, Visser SN, White CP 2003: Attention-deficit/hyperactivity disorder in school-aged children: association with maternal mental health and use of health care resources. Pediatrics, 111:1232-7.

Lim HK, Hubbard JW, Midha KK. 1986. Development of enantioselective gas chromatographic quantitation assay for d.1-threo-methylphenidate in biological fluids. J Chromatography, A 378:109-23.
Logan J, Fowler JS, Volkow ND, et al. 2001. A strategy for removing the bias in the graphical analysis method. J Cereb Blood Flow Metab, 21:307-20.

Logan J, Fowler JS, Volkow ND, et al. 1996. Distribution volume ratios without blood sampling from graphical analysis of PET data. J Cereb Blood Flow Metab, 16:834-40.

Markowitz JS, Straughn AB, Patrick KS, 2003. Advances in the pharmacotherapy of attention-deficit-hyper activity disorder: focus on methylphenidate formulations. Pharmacotherapy, 23:1281-99.

Markowitz JS, DeVane CL, Pestreich L, et al. 2005. Differentiation of d,L- and dl-methylphenidate through in vitro pharmacological screening. Proceedings of Abstracts from Oral and Poster Presentations, NCDEUNIMH 45th Annual Meeting; Boca Raton, FL, USA. p 186.

Modi NB, Wang B, Noveck RJ, et al. 2000. Gupta SK. Dose-proportional and stereospecific pharmacokinetics of methylphenidate delivered using an osmotic, controlled-release oral delivery system. J Clin Pharmacol, 40:1141-9.

Patrick KS, Caldwell RW, Ferris RM, et al. 1987. Pharmacology of the enantiomers of threo-methylphenidate. J Pharmacol Exp Ther, 241:152-8.

Patrick KS, Gonzalez MA, Straughn AB, et al. 2005. New methylphenidate formulations for the treatment of attention-deficit/hyperactivity disorder. Expert Opin Drug Deliv, 2:121-43.

Patrick KS, Kilts CD, Breese GR. 1981. Synthesis and pharmacology of hydroxylated metabolites of methylphenidate. J Med Chem, 29:1237-40.

Novartis Pharmaceuticals Corporation. 2005. PDR Focalin XR, Insert package, Novartis Pharmaceuticals Corporation, East Hanover, New Jersey, USA.

Quinn DM. 2000. Methylphenidate: the role of d-isomer. In Ritalin. Theory and practice. 2nd ed. New York: MA Liberty Publishers Larchmont. p 369-74.

Quinn D, Wigal S, Swanson J, et al. 2004. Comparative pharmacodynamics and plasma concentrations of d-threo-methylphenidate hydrochloride after single doses of d-threo-methylphenidate hydrochloride and d,l-threo-methylphenidate hydrochloride in a double-blind, placebocontrolled, crossover laboratory school study in children with attentiondeficit/hyperactivity disorder. J Am Acad Child Adolesc Psychiatry, 43:1422-9.

Scheffler MR, Teo S, Stirling D, et al. 2003. Multiple dosing of dexmethylphenidate HLC (d-MPH) does not result in accumulation or interconversion tp 1-methylphenidate HCL in male and female children. Clin Pharmacol Ther, II:27.

Silva R, Tilker HA, Cecil JT, et al. 2004. Open-label study of dexmethylphenidate hydrochloride in children and adolescents with attention deficit hyperactivity disorder. J Child Adolesc Psychopharmacol, 14:555-63.

Silva, RR, Muniz, R, Pestreich, L, et al. 2005 Performance improvement in school children with ADHD receiving once-daily dexmethylphenidate. Scientific Proceedings from the American Academy of Child and Adolescent Psychiatry 52nd Annual Meeting. Toronto, Canada. p 121.

Silva RR, Muniz R, Pestreich L, et al. 2006. Efficacy and duration of effect of extended-release dexmethylphenidate vs placebo in school children with attention-deficit/hyperactivity disorder. J Child Adolesc Psychopharmacol. In press.

Srinivas NR, Hubbard JW, Quinn D, et al. 1992. Enantioselective pharmacokinetics and pharmacodynamics of d, 1-threo-methylphenidate in children with attention deficit hyperactivity disorder. Clin Pharmacol Ther, 52:561-8.

Srinivas NR, Quinn D, Hubbard JW, et al. 1987. Stereoselective disposition of methylphenidate in children with attention-deficit disorder. $J$ Pharmacol Exp Ther, 241:300-6.

Srinivas NR, Hubbard JW, Koechinsk ED, et al.1993. Enantioselective pharmacokinetics of d.l-threo-methylphenidate in humans. Pharm Res, 10:14-21.

Sun Z, Murry DJ, Sanghani SP, et al. 2004. Methylphenidate is stereoselectively hydrolyzed by human carboxylesterase CES1A1. J Pharmacol Exp Ther, 310:469-76. 
Swanson JM, Lerner M, Wigal T, et al.2002. The use of a laboratory school protocol to evaluate concepts about efficacy and side effects of new formulations of stimulant medications. J Atten Disord, 6(Suppl 1): S73-88.

Teo SK, Stirling D, Thomas S, et al. 2002. A 90-day oral gavage toxicity study of D-methylphenidate and D, L-methylphenidate in SpragueDawley rats. Toxicology, 179:183-96.

Teo SK, San RH, Wagner VO, et al. 2003a. D-Methylphenidate is nongenotoxic in in vitro and in vivo assays. Mutation Res, 537:67-79.

Teo SK, Scheffler MR, Wu A, et al. 2004. A single-dose, two-way crossover, bioequivalence study of dexmethylphenidate $\mathrm{HCl}$ with and without food in healthy subjects. $J$ Clin Pharmacol, 44:173-8.

Teo SK, Stirling DI, Hoberman AM, et al. 2003b. D-methylphenidate and D,L-methylphenidate are not developmental toxicants in rats and rabbits. Birth Defects Res B Dev Reprod Toxicol, 68:162-71.

Teo SK, Stirling DI, Thomas SD, et al. 2003c. A 90-day oral gavage toxicity study of d-methylphenidate and d,l-methylphenidate in beagle dogs. Inter J Toxicol, 22:215-26.

Teo SK, Stirling DI, Thomas SD, et al. 2002. The perinatal and postnatal toxicity of D-methylphenidate and D,L-methylphenidate in rats. Reprod Toxicol, 6:353-66.

Teo SK, Stirling DI, Thomas SD, et al. 2003d. Neurobehavioral effects of racemic threo-methylphenidate and its $\mathrm{D}$ and $\mathrm{L}$ enantiomers in rats. Pharmacol Biochem Behav, 74:747-54.

Volkow ND, Chang L, Wang GJ, et al. 2001a. Loss of dopamine transporters in methamphetamine abusers recovers with protracted abstinence. J Neuroscience, 21:9414-8.
Volkow ND, Chang L, Wang GJ, et al. 2001b. Association of dopamine transporter reduction with psychomotor impairment in methamphetamine abusers. Am J Psychiatry, 158:377-82.

Volkow ND, Ding YS, Fowler JS, et al. 1996. Dopamine transporters decrease with age. $J$ Nucl Med, 37:554-9.

Volkow ND, Ding YS, Fowler JS, et al. 1995. A new PET ligand for the dopamine transporter: studies in the human brain. $J$ Nucl Med, $36: 2162-8$.

Volkow ND, Fowler JS, Wang G, et al. 2002. Mechanism of action of methylphenidate: insights from PET imaging studies. J Atten Disord, 6(Suppl):S31-43.

Wang GJ, Volkow ND, Fowler JS, et al. 1995. Comparison of two pet radioligands for imaging extrastriatal dopamine transporters in human brain. Life Sci, 57:187-91.

Weiss M, Wasdell M, Patin J. 2004. A post hoc analysis of d-threomethylphenidate hydrochloride (focalin) vs d,l-threo-methylphenidate hydrochloride (ritalin). J Am Acad Child Adolesc Psychiatry, 43:1415-21.

Wigal S, Swanson JM, Feifel D, et al. 2004. A double-blind, placebo-controlled trial of dexmethylphenidate hydrochloride and d,1-threo-methylphenidate hydrochloride in children with attention-deficit/hyperactivity disorder. $J$ Am Acad Child Adolesc Psychiatry, 43:1406-14.

Wilens TE, Faraone SV, Biederman J. 2004. Attention-deficit/hyperactivity disorder in adults. JAMA, 292:619-23. 
